\title{
ZEROS AND FACTORIZATIONS OF HOLOMORPHIC FUNCTIONS
}

\author{
BY WALTER RUDIN ${ }^{1}$
}

Communicated by H. Helson, May 31, 1966

For $N=1,2,3, \cdots$ we let $U^{N}$ denote the Cartesian product of $N$ copies of the open unit disc $U$. I.e., $U^{N}$ consists of all $z=\left(z_{1}, \cdots, z_{N}\right)$ in $C^{N}$ (the space of $N$ complex variables) with $\left|z_{j}\right|<1$ for $j=1, \cdots, N$. We write $U$ in place of $U^{1}$. If $1 \leqq p<\infty, H^{p}\left(U^{N}\right)$ is the space of all holomorphic functions $f$ in $U^{N}$ for which

$$
\sup (1 / 2 \pi)^{N} \int_{-\pi}^{\pi} \cdots \int_{-\pi}^{\pi}\left|f\left(r_{1} e^{i \theta_{1}}, \cdots, r_{N} e^{i \theta_{N}}\right)\right|{ }^{p} d \theta_{1} \cdots d \theta_{N}<\infty,
$$

the supremum being taken over all choices of $r_{1}, \cdots, r_{N}$ such that $0 \leqq r_{j}<1$. The $p$ th root of this supremum is defined to be $\|f\|_{p}$; this gives a Banach space norm. (The boundary behavior of these functions is discussed in Chapter XVII of [3].)

The class of all bounded holomorphic functions in $U^{N}$ is denoted by $H^{\infty}\left(U^{N}\right)$.

The zero-set of a function $f$ defined in $U^{N}$ is the set of all $z \in U^{N}$ at which $f(z)=0$.

It is well known that the zero-set of every $f \in H^{p}(U)$, for any $p$, is also the zero-set of some $g \in H^{\infty}(U)$. These zero-sets, in one variable, are completely characterized by the Blaschke condition $\sum\left(1-\left|\alpha_{i}\right|\right)$ $<\infty$. For $N>1$ a different phenomenon occurs:

Theorem A. There exists a function $f$, not identically 0 , such that

(a) $f \in H^{p}\left(U^{2}\right)$ for all $p<\infty$, but

(b) if $g \in H^{\infty}\left(U^{2}\right)$ and if the zero-set of $g$ contains the zero-set of $f$, then $\mathrm{g}$ is identically 0 .

Let us call a subspace $S$ of $H^{p}\left(U^{N}\right)$ invariant if multiplication by the coordinate functions $z_{1}, \cdots, z_{N}$ maps $S$ into $S$. The closed invariant subspaces of $H^{p}(U)$ are known precisely: they are generated by inner functions $[1, \mathrm{pp} .8,25]$. But if we consider the smallest closed invariant subspace of $H^{p}\left(U^{2}\right)$ which contains the function $f$ of Theorem A we obtain the following:

CoROllary. If $1 \leqq p<\infty$, there is a nontrivial closed invariant subspace of $H^{p}\left(U^{2}\right)$ which contains no bounded function (except 0 ).

\footnotetext{
${ }^{1}$ Supported by NSF Grant GP--3483.
} 
To every $f \in H^{1}(U)$ there correspond two functions $g, h \in H^{2}(U)$ such that $f \in g h$. The usual proof of this factorization theorem [3, Vol. I, p. 275] even shows that $g$ and $h$ can be so constructed that their boundary values satisfy $|g|^{2}=|h|^{2}=|f|$ a.e. The work of Helson and Lowdenslager has extended this stronger result to $H^{1}$-functions on compact connected abelian groups $G$, where analyticity is defined relative to some total order of the dual group of $G$ [2, p. 208]. It seems likely that the factorization fails in $H^{1}\left(U^{N}\right)$ if $N>1$. The following theorem shows at least that the above-mentioned stronger result fails very badly if $N>2$.

Theorem B. Suppose $\epsilon>0, M<\infty$. There exists an irreducible homogeneous polynomial $f$ in 3 variables, with $\|f\|_{1}<\epsilon,\|f\|_{2}>M$. For any such $f$ we have $\|g\|_{2}\|h\|_{2}>M$ whenever $f=g h$ and $g, h \in H^{2}\left(U^{3}\right)$.

An immediate consequence of Theorem $B$ is the observation that the bilinear continuous map

$$
\mu: H^{2}\left(U^{3}\right) \times H^{2}\left(U^{3}\right) \rightarrow H^{1}\left(U^{3}\right),
$$

defined by $\mu(g, h)=g h$, is not open at the origin. This by itself may imply that the range of $\mu$ cannot be all of $H^{1}\left(U^{3}\right)$.

In any case, Theorem $B$ suffices to establish a "nonfactorization theorem" for Dirichlet series. Let us say that a function $F$ of the form

$$
F(s)=\sum_{n=1}^{\infty} a_{n} n^{-s}
$$

is a Dirichlet series of class $H^{p}(1 \leqq p<\infty)$ if (a) the series converges absolutely in the open right half-plane, and (b)

$$
\sup _{\sigma>0}\left\{\lim _{T \rightarrow \infty} 1 / 2 T \int_{-T}^{T}|F(\sigma+i t)|^{p} d t\right\}<\infty .
$$

(The existence of the limit is assured by almost periodicity.)

Theorem C. There exists a Dirichlet series of class $H^{1}$ which is not a product of two Dirichlet series of class $\mathrm{H}^{2}$.

We conclude with brief outlines of the proofs.

Proof of Theorem A. Fix a number $R>1$. Let $\left\{\alpha_{k}\right\}$ be a sequence of complex numbers, $\left|\alpha_{k}\right|=1$, such that every point of some infinite set $E$ occurs infinitely many times in $\left\{\alpha_{k}\right\}$. Let $\left\{n_{k}\right\}$ be a rapidly increasing sequence of positive integers. Define

$$
f(z, w)=\prod_{k=1}^{\infty}\left\{1-R\left(\frac{z+\bar{\alpha}_{k} w}{2}\right)^{n k}\right\} .
$$


Note that $\left|z+\bar{\alpha}_{k} w\right| \leqq 2$ on the closure of $U^{2}$ and that equality occurs only on a circle in the distinguished boundary $T^{2}$, i.e., on a set of measure 0 . Hence $\left\{n_{k}\right\}$ can be chosen inductively so that the integrals $\int_{T^{2}}\left|f_{m}\right|^{p}$ are bounded, as $m \rightarrow \infty$, for each $p<\infty$ (the bound will depend on $p$ ), where $f_{m}$ denotes the product of the first $m$ factors. The product will also converge uniformly on compact subsets of $U^{2}$. Thus $f \in H^{p}\left(U^{2}\right)$ and $f$ does not vanish identically.

Suppose $g \in H^{\infty}\left(U^{2}\right)$ and $g=0$ whenever $h=0$. For $\beta \in E$ and $\lambda \in U$ put $g_{\beta}(\lambda)=g(\lambda, \beta \lambda)$. If $\alpha_{k}=\beta$, then $g_{\beta}$ vanishes at every $n_{k}$ th root of $1 / R$. This happens for infinitely many $k$, and since $g_{\theta} \in H^{\infty}(U)$ one deduces from Jensen's formula that $g_{\beta}(\lambda)=0$ for all $\lambda \in U$. In other words, the zero-set of $g$ contains every disc

$$
D_{\beta}=\{(\lambda, \beta \lambda): \lambda \in U\} \quad(\beta \in E) .
$$

All these discs intersect at $(0,0)$. This forces $g$ to be identically 0 .

Proof of Theorem B. Let $P_{n}$ be the space of all homogeneous polynomials of degree $n$, in 3 variables. If $n$ is large enough, there exists $f \in P_{n}$ with $\|f\|_{1}<\epsilon,\|f\|_{2}>M$. A dimensionality argument shows that the irreducible members of $P_{n}$ form a dense (in fact, open) subset of $P_{n}$. Hence we can adjust $f$ so that it is irreducible. If now $f=g h$, $g=\sum g_{k}, h=\sum h_{k}$, where $g_{k}$ and $h_{k}$ are homogeneous polynomials of degree $k$, then $f$ is the product of the lowest nonvanishing components of $g$ and $h$, say $f=g_{j} h_{n-j}$. But $f$ is irreducible. Hence $j=0$ or $j=n$. Finally, $\|g\|_{2} \geqq\left\|g_{j}\right\|_{2}$, since the various $g_{k}$ 's are orthogonal to each other; likewise, $\|h\|_{2} \geqq\left\|h_{n-j}\right\|_{2}$.

Proof of Theorem C. There are homogeneous irreducible polynomials $f_{k}\left(z_{1}, z_{2}, z_{3}\right)$ with $\left\|f_{k}\right\|_{1}<2^{-k},\left\|f_{k}\right\|_{2}>k$. Let $N_{k}$ be the degree of $f_{k}$, let $C_{k}$ be the sum of the absolute values of the coefficients of $f_{k}$, let $\left\{p_{j}\right\}$ be an increasing sequence of distinct primes such that

$$
p_{3 k}>\left(k^{2} C_{k}\right)^{k / N_{k}},
$$

and define

$$
F(s)=\sum_{k=1}^{\infty} f_{k}\left(\overrightarrow{p_{3 k}}, \overrightarrow{p_{3 k+1}}, \overrightarrow{p_{3 k+2}}\right) .
$$

Our choice of $\left\{p_{j}\right\}$ assures the absolute convergence of the Dirichlet series of $F(s)$, if $\operatorname{Re} s>0$. With the aid of Theorem B it follows easily that this $F$ has the properties stated in Theorem C. In fact, one can even show that $F$ is not the product of any finite number of Dirichlet series of class $H^{2}$. 


\section{REFERENCES}

1. Henry Helson, Lectures on invariant subspaces, Academic Press, New York, 1964.

2. Walter Rudin, Fourier analysis on groups, Interscience, New York, 1962.

3. Antoni Zygmund, Trigonometric series, 2nd ed., Cambridge Univ. Press, New York, 1959.

UNIVERSITY OF WisCONSIN 